\title{
Dynamic assessment of punctate white matter damage in premature infants based on conventional MRI and apparent diffusion coefficient value
}

\section{Jiping Zhao}

Affiliated Hospital of Medical College Qingdao University

Chunxiao Cui

Affiliated Hospital of Medical College Qingdao University

Haozhi Lin

Affiliated Hospital of Medical College Qingdao University

Minge Ma

Affiliated Hospital of Medical College Qingdao University

Chongfeng Duan

Affiliated Hospital of Medical College Qingdao University

Xuejun Liu ( $\boldsymbol{\sigma}$ bncz@sina.com )

Affiliated Hospital of Medical College Qingdao University

https://orcid.org/0000-0002-3250-4778

Research article

Keywords: Magnetic resonance imaging; white matter damage; premature infants; apparent diffusion coefficient

Posted Date: January 28th, 2020

DOI: https://doi.org/10.21203/rs.2.22010/v1

License: (9) This work is licensed under a Creative Commons Attribution 4.0 International License.

Read Full License 


\section{Abstract}

Background The purpose of this study was to observe the distribution of punctate white matter damage (PWMD) in premature infants and the signal characteristics of conventional and diffusion weighted imaging MRI .To explore the application value of routine MRI and ADCvalue in evaluating white matter damage in premature infants. Methods $A$ total of 218 premature infants were enrolled from the Affiliated Hospital of Qingdao University and divided into a case group $(n=110)$ and a control group $(n=108)$. ADC values of lesions in white matter, areas around lesions (within $5 \mathrm{~mm}$ ) and contralateral areas of mirror images were measured. ADC values of the same areas were reexamined and measured after 5-7 weeks. ADC values were measured in normal appearing areas of the case group and control group. Results 1. A total of 390 white matter lesions were found in 110 children in the case group.324 lesions (83.1\%) had a hyperintense signal in T1WI, 352 (90.3\%) had a hyperintense signal in diffusion weighted imaging (DWI), 326 (83.5\%) had a hyperintense signal in fluid-attenuated inversion recovery (FLAIR), and 118 (30.3\%) had a low signal on T2WI. 2. The ADC value in the lesion was significantly lower than that in the surrounding area and the contralateral area, and the ADC value in the surrounding area was also lower than that in the contralateral area. At the time of reexamination, the ADC value of the lesions was still significantly lower than that of the surrounding and contralateral areas of the lesion. In the case group, the ADC values of the normal area in the bilateral centrum ovale and in the splenium of the corpus callosum were lower than those in the control group. Conclusion In the early stage, (within 7 days), DWI was the most sensitive to detecting lesions, and the detection rate of lesions on T1WI was the highest as time went on. By measuring the $A D C$ value, we can find the damage in the surrounding area of the lesion and the damage in the normal area $₫$ which can not be detected on a conventional MRI sequence.

\section{Background}

In recent years, the incidence of premature infants domestic and abroad has increased annually [1-3]. With the progress of diagnosis and treatment, the survival rate of critically premature infants has been significantly improved, but serious neurological sequelae of some children have caused serious impacts on society and their families, and white matter damage is one of the main forms of damage. The prognosis of diffuse white matter damage is poor, and the incidence is relatively low, while the incidence of punctate white matter damage is high, which is the main type of white matter damage in premature infants. At present, MRI has become the main diagnostic method of white matter damage, and DWI can detect the occurrence of white matter damage earlier than conventional MRI [5] and can detect cytotoxic brain edema caused by white matter damage. However, the T2 transmission effect of DWI may lead to a false-positive diagnosis. The diagnostic physicians can only use the naked eye to judge the damaged area and normal area on the DWI sequence, so there will inevitably be errors among different physicians. The ADC value can quantitatively analyze the diffusion ability of water molecules in lesions to quantitatively assess the degree and extent of white matter damage. When white matter damage occurs, the diffusion of water molecules is limited, and the ADC value of lesions decreases [6-7]. In this study, we 
mainly discussed the signal and distribution characteristics of white matter damage in premature infants and the changes in $A D C$ values in lesion and normal white matter areas.

\section{Methods}

\subsection{Research objective}

We enrolled premature infants born in the Affiliated Hospital of Qingdao University from October 2015 to May 2019 .Premature infants with gestational age less than 34 weeks or body weight less than $2 \mathrm{~kg}$ were routinely examined by MRI. Premature infants whose mothers have high risk factors, such as hypertension, diabetes, infection and so on, should also undergo MRI. Cranial magnetic resonance examination (including T1WI, T2WI, FLAIR, DWI) was completed within 10 days after birth. The MR images of each patient were evaluated by three doctors.A total of 110 premature infants with white matter damage on the conventional MR sequencewere selected as the case group $(n=110)$, and 108 premature infants with normal performance on the conventional MR sequence were selected as the control group. There were 64 males and 46 females in the case group, with an average gestational age of $33.6+1.3$ weeks. There were 66 males and 42 females in the control group, with an average gestational age of $34+1.6$ weeks. There was no difference in gender, examination time or gestational age between the two groups $(P>0.05)$.

\subsection{MRI scanning}

All subjects completed routine MRI examinations within 10 days after birth, and their ADC values were measured. Five to 7 weeks later, the patients with PWMD were reexamined by routine MRI examination (average age $42 \pm 6$ days), and the ADC values of the corresponding sites were measured again.

Scanning parameters and equipment include the GE3.0T magnetic resonance instrument, and the scanning parameters were as follows:T1WI (TR: $1708 \mathrm{~ms}$, TE: $10 \mathrm{~ms}$, including sagittal and axial scanning, slice thickness: $4 \mathrm{~mm}$, slice gap $0.5 \mathrm{~mm}$, NEX: 2).T2WI (TR: $3580 \mathrm{~ms}$, TE: $104 \mathrm{~ms}$, axis scanning, slice thickness: $4 \mathrm{~mm}$, slice gap: $0.5 \mathrm{~mm}$, NEX: 2).FLAIR (8402 ms, TE: $131 \mathrm{~ms}$, axis scanning, 4 $\mathrm{mm}$, slice thickness: $0.5 \mathrm{~mm}$ slice gap, NEX: 2).DWI (EPI-SE, TR=2120 ms, TE=63 ms, b values were 0 and $1000 \mathrm{~s} / \mathrm{mm}$.axis scanning, slice thickness: $4 \mathrm{~mm}$, slice gap:0.5 mm).

\subsection{Measurement of ADC values}

The DWI image was converted to an ADC image by Functiontool software of GE magnetic resonance workstation. The ADC value of the corresponding area was measured by three experienced physicians and the average value was taken.

Case group: The ADC value of each lesion was measured at the first MRI scan, and its average value was calculated as the $A D C$ value of the lesion area. The ADC value of three points was measured in the range of $5 \mathrm{~mm}$ around each lesion, and then the average value was calculated as the ADC value around the lesion. The ADC value of the normal area of the contralateral cerebral hemisphere was measured in each 
lesion, and the average value was taken as the ADC value of the contralateral region. The ADC values of the above parts were measured according to the same method when MRI results were reexamined. The $A D C$ values were measured at three points in the bilateral centrum ovale, bilateral corona radiata, bilateral optic radiation, splenium of the corpus callosum and posterior limb of the internal capsule. Then, the average $A D C$ value was taken as the $A D C$ value of the bilateral centrum ovale, bilateral corona radiata, bilateral optic radiation, splenium of the corpus callosum and posterior limb of the internal capsule.

Control group: The ADC values were measured at three points in the bilateral centrum ovale, bilateral corona radiata, bilateral optic radiation, splenium of the corpus callosum and posterior limb of the internal capsule. Then, the average ADC value was taken as the ADC value of the bilateral centrum ovale, bilateral corona radiata, bilateral optic radiation, splenium of the corpus callosum and posterior limb of the internal capsule.

\subsection{Statistical methods}

SPSS 19.0, independent sample T-test, paired sample T-test, and chi-square test were used. A P $<0.05$ was considered statistically significant.

Fig 1, 2 As shown by the black arrow, the lesions showed a hyperintense signal in DWI and a decreased signal on $A D C$, and the range of some lesions on ADC was slightly larger than that on DWI (as shown by the white arrow); Fig 3-5 The lesions showed a hyperintense signal in T1WI and FLAIR and a low signal on T2WI (indicated by the black arrow); Fig 6, 7 and 8 The abnormal signal on DWI, ADC and T1WI disappeared at the time of reexamination; Fig 9 The ADC value in the focus (white circle) of a child was $1.08 \times 10^{-3} \mathrm{~mm} 2 / \mathrm{s}$, the average $A D C$ value of three points (red circle) in the $5 \mathrm{~mm}$ range (black circle) around the lesion was $1.58 \times 10^{-3} \mathrm{~mm} 2 / \mathrm{s}$, and the $A D C$ value in the contralateral area of the lesion was $1.72 \times 10^{-3} \mathrm{~mm} 2 / \mathrm{s}$.

\section{Results}

\subsection{Distribution characteristics of lesions}

A total of 390 lesions were found in 110 children with white matter damage, and their distribution in the white matter is shown in Table 1. There were $315(80.8 \%)$ lesions in the central area (bilateral optic radiation, corona radiata I area, centrum ovale, paraventricular white matter, posterior limb of internal capsule, splenium of corpus callosum). There were 75 lesions (19.2\%) in the peripheral area (bilateral frontal-parietal-temporal-occipital lobe white matter).

Table 1 Distribution of lesions in different brain regions of the case group 


\begin{tabular}{|c|c|c|}
\hline Position & Number of lesions & Percentage \\
\hline Apparent radiation & 114 & $29.2 \%$ \\
\hline Corona radiata & 116 & $29.7 \%$ \\
\hline Centrum ovale & 35 & $9 \%$ \\
\hline Parasomal white matter of lateral ventricle & 14 & $3.6 \%$ \\
\hline White matter of frontal lobe & 25 & $6.4 \%$ \\
\hline White matter of parietal lobe & 28 & $7.2 \%$ \\
\hline White matter of temporal lobe & 9 & $2.3 \%$ \\
\hline White matter of occipital lobe & 13 & $3.3 \%$ \\
\hline Paraventricular white matter & 10 & $2.6 \%$ \\
\hline Posterior limb of internal capsule & 6 & $1.5 \%$ \\
\hline Paraventricular posterior horn & 18 & $4.6 \%$ \\
\hline Splenium of corpus callosum & 2 & $0.5 \%$ \\
\hline Total & 390 & $100 \%$ \\
\hline
\end{tabular}

\subsection{Signal characteristics of lesions}

The average age at the initial examination was 7 days. The signal characteristics of 390 lesions in 110 children are shown in Table 2. Among them, there were 286 lesions (73.3\%) with a hyperintense signal in both T1WI and DWI, 38 lesions (9.7\%) with a hyperintense signal in T1WI and an equal signal on DWI, 66 lesions (16.9\%) with a hyperintense signal in DWI and an equal signal on T1WI. Seventy-two children were scanned within 7 days after birth, with a total of 262 lesions. Thirty-eight children were scanned within 7 to 10 days after birth, with a total of 128 lesions (Table 2). The detection rate of lesions on the DWI sequence was the highest in children examined within 7 days after birth, and the detection rate on the T1WI sequence was the highest in children examined more than 7 days after birth. The chi-square test showed that the differences were statistically significant (Tables 3 and 4). At the time of reexamination, 352 hyperintense lesions on the DWI sequence disappeared (100\%), and 314 of 324 hyperintense lesions on the T1WI sequence disappeared (96.9\%). 
Table 2 Number of abnormal signals on different sequences at the initial examination (number, percentage of lesions)

\begin{tabular}{|c|c|c|c|c|c|c|}
\hline $\begin{array}{l}\text { Inspection } \\
\text { time }\end{array}$ & $\begin{array}{l}\text { Number of } \\
\text { children }\end{array}$ & $\begin{array}{c}\text { Number of } \\
\text { lesions }\end{array}$ & $\begin{array}{l}\text { Hyperintense } \\
\text { signal in T1WI }\end{array}$ & $\begin{array}{c}\text { Low signal in } \\
\text { T2WI }\end{array}$ & $\begin{array}{c}\text { Hyperintense signal } \\
\text { in FLAIR }\end{array}$ & $\begin{array}{l}\text { Hyperintense } \\
\text { signal in DWI }\end{array}$ \\
\hline $\begin{array}{c}\text { Within } 7 \\
\text { days }\end{array}$ & 72 & 262 & $204(77.9 \%)$ & $48(18.3 \%)$ & $220(84 \%)$ & 246(93.9\%) \\
\hline $\begin{array}{l}\text { More than } \\
7 \text { days }\end{array}$ & 38 & 128 & $120(93.8 \%)$ & $70(54.7 \%)$ & $106(82.8 \%)$ & $106(82.8 \%)$ \\
\hline Total & 110 & 390 & $324(83.1 \%)$ & $118(30.3 \%)$ & $326(83.5 \%)$ & $352(90.3 \%)$ \\
\hline
\end{tabular}

Table 3 Chi-square test for the number of abnormal signals on T1WI, FLAIR and DWI sequences in children examined within 7 days of birth

\begin{tabular}{|c|c|c|c|c|}
\hline & & \multicolumn{2}{|c|}{ Signal } & \multirow[b]{2}{*}{ Total } \\
\hline & & Hyperintens & atens & \\
\hline \multirow[t]{3}{*}{ Magnetic resonance sequences } & T1WI & 204 & 58 & 262 \\
\hline & FLAIR & 220 & 42 & 262 \\
\hline & DWI & 246 & 16 & 262 \\
\hline Total & & 670 & 116 & 786 \\
\hline
\end{tabular}

There was no significant difference between T1WI and FLAIR sequence $(P=0.75)$. There was significant difference between DWI and T1WI sequence $(\mathrm{P}<0.001)$.There was significant difference between DWI and FLAIR sequence $(\mathrm{P}<$ $0.001)$. 
Table 4 Chi-square test for the number of abnormal signals on T1WI, FLAIR and DWI sequences in children examined 7 days after birth

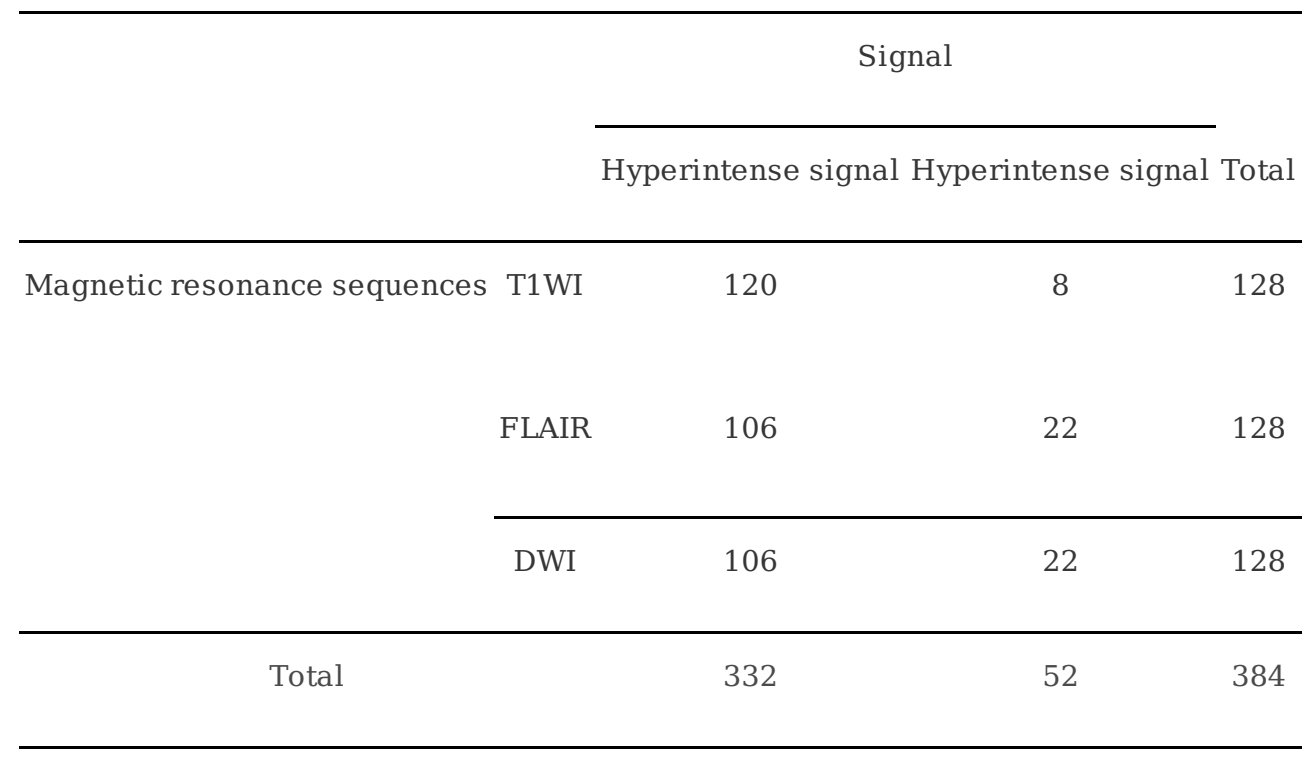

Comparing T1WI with FLAIR and DWI sequences, the difference was statistically significant $\mathrm{P}=0.006$.

\subsection{ADC value measurement}

\subsection{Comparison of ADC values in lesion area, surrounding lesion and mirrored contralateral region}

The ADC value of the lesion area was significantly lower than that of the surrounding area and the contralateral area of the lesion (Table 5), and a T-test of paired samples showed that the difference was statistically significant $(P<0.01)$. The $A D C$ value of the surrounding area was lower than that of the contralateral area, and a T-test of paired samples showed a significant difference $(P=0.021)$.

At the time of reexamination, the $A D C$ value in the lesion area increased significantly, while the ADC value in the surrounding and contralateral areas decreased. The differences were statistically significant. The $A D C$ value in the area around the lesion was still significantly lower than that in the surrounding area and the contralateral area. The T-test of paired samples showed a significant difference $(P<0.01)$; the ADC value in the area around the lesion was also lower than that in the contralateral area, and the T-test of paired samples showed a significant difference $(P=0.015)$.

Table 5 The apparent diffusion coefficients $\left(\times 10^{-3} \mathrm{~mm}^{2} / \mathrm{s}\right.$, ’x $\left.\pm \mathrm{s}\right)$ of lesions, surrounding lesions and contralateral area, and P values of differences between the two scans 


\begin{tabular}{lccc}
\hline & Lesion area & Perilesional area & Contralateral area \\
\hline First scans & $1.049+0.246$ & $1.532+0.201$ & $1.571+0.178$ \\
\hline Review & $1.408+0.189$ & $1.444+0.204$ & $1.466+0.220$ \\
\hline P value & 0.0001 & 0.0001 & 0.0001 \\
\hline
\end{tabular}

\subsection{Comparison of ADC values between the normal area of the case group and the control group}

The ADC values of the normal area, bilateral centrum ovale and splenium of corpus callosum in the case group were lower than those in the control group (Table 6). Independent sample T tests showed that the differences were statistically significant $(P=0.01 ; P=0.003)$.

Table 6 The apparent diffusion coefficients $\left(\times 10^{-3} \mathrm{~mm}^{2} / \mathrm{s}\right.$, ' $\left.\mathrm{x} \pm \mathrm{s}\right)$ in normal areas of the case group and the control group, and the $\mathrm{P}$ values between the two groups

Centrum ovale Corona radiata Apparent radiation Splenium of corpus callosum Posterior limb of internal capsule

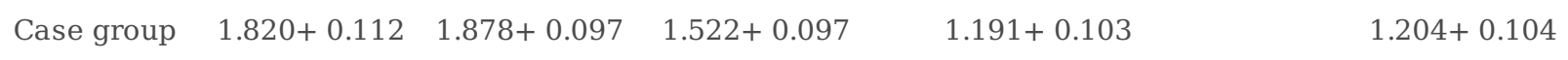

\begin{tabular}{lllll}
\hline control group $1.854+0.076$ & $1.861+0.088$ & $1.531+0.080$ & $1.232+0.096$ & $1.212+0.080$
\end{tabular}

\begin{tabular}{lllll}
\hline P value & 0.010 & 0.16 & 0.468 & 0.003
\end{tabular}

\section{Discussion}

With the increasing application of MRI in premature infants, reports of punctate white matter damage in premature infants are increasing year by year. The main manifestations of punctate white matter damage were dotted, linear and clustered hyperintense signals in DWI. Over time, a hyperintense signal in T1WI appeared in the corresponding parts, with or without low signals on T2WI [8]. Most of the abnormal signals disappeared after 3 to 4 weeks of birth, and a small number of children eventually developed periventricular leukomalacia (PVL) [9]. Because the brain of premature infants is still immature, it is susceptible to various risk factors. Moreover, the metabolism of deep white matter is higher, and the density of cerebrovascular matter decreases temporarily at 28 to 36 weeks of gestation, so white matter is more vulnerable to damage in this period [10].

\subsection{Distribution of lesions and characteristics of MRI signal}


Punctate white matter damage in premature infants occurs mainly in the bilateral centrum ovale, bilateral corona radiata and bilateral periventricular areas. This study showed that most of the white matter damage was located in the bilateral corona radiata (29.7\%), followed by bilateral optic radiation (29.2\%), and the focus in the central area accounted for $80.8 \%$. Comette et al. [11] showed that white matter damage in premature infants often distributes around the lateral ventricles and is located in the central region, which was consistent with the results of this study. Roelants [12] et al. showed that the glutamic compound increased significantly in the lesion area within 1 week of birth, leading to mast cell phagocytosis and gliocyte hyperplasia, thus reducing the water content in surrounding tissues and shortening the relaxation time of T1 and T2. Gomori [13] et al. believed that this signal change was caused by the decomposition products of red blood cells, but magnetic susceptibility-weighted imaging showed that there were no microhemorrhagic components in the lesions [14]. This study showed that DWI is more sensitive to lesion detection in the early stage, and over time, T1WI is more sensitive to lesion detection. Miller [15] et al. showed that the hyperintense signal in DWI indicates that the diffusion of intracellular water molecules is limited, so the lesions could be detected in the early stage, and this study showed that the detection rate of lesions by DWI is the highest within one week after birth. FLAIR is an important diagnostic index for punctate white matter damage because it has a high detection level and is similar to $\mathrm{T} 1 \mathrm{WI}$. The detection rate of $\mathrm{T} 2 \mathrm{WI}$ is low in each period, so it can only be used as an auxiliary reference index.

\subsection{Measurement of ADC value}

The ADC value of the lesion was significantly lower than that of the surrounding area and the contralateral area. The ADC value of the lesion decreased significantly, suggesting that the pathological mechanism of the lesion was cytotoxic edema. Inder [16] et al. showed that children with bilateral paraventricular diffuse white matter damage showed symmetrical hyperintense signals in DWI, and the $A D C$ value was lower than that of normal preterm infants of the same gestational age. Feldman [17] et al. found that the ADC value of children with white matter damage was significantly lower than that of normal newborns. The study by Tong Xin [18] et al. also showed that the ADC value of the brain white matter damaged area was significantly lower than that of surrounding areas and lower than that of normal premature infants. Their results were consistent with this study. However, their results showed no difference in ADC values between the surrounding area of the lesion and normal preterm infants. Although no abnormal signal was found in the surrounding area of the lesion in conventional sequence and DWI in this study, the ADC value of the surrounding area was lower than that of the contralateral areas of the lesion, suggesting that the damage range of white matter may be larger than that shown in conventional sequences. The area around the lesion we measured was only within $5 \mathrm{~mm}$ of the lesion, and the extent of white matter damage around the lesions remains to be further studied. Marin-Padilla [19] et al. found abnormal neurons in the cortex around the lesion by special pathological methods, which may be caused by white matter damage that affects the differentiation of cortical neurons.

This study showed that the ADC values in the normal areas of coronal radiata and splenium of corpus callosum in children with white matter damage were lower than those in normal preterm infants. 
According to the results, it can be inferred that mild white matter damage may also occur in normal areas of conventional MRI. The structural integrity of white matter determines the neurobehavioral ability at the later stage [20], which is why many premature infants with mild white matter damage on early MRI scans show significant cognitive impairment at school age.

At the time of reexamination, the hyperintense signal in DWI of all lesions disappeared, and the ADC value increased, indicating that the lesions were recovering. The ADC values of the surrounding and contralateral areas decreased, indicating that the white matter of the brain was in the process of myelination. However, the ADC value in the lesion area is still significantly lower than that in the surrounding area and the contralateral area, and the ADC value in the surrounding area is also lower than that in the contralateral area, suggesting that the disappearance of abnormal signal in DWI does not mean the complete recovery of water molecular diffusion disorder. At this time, white matter damage may still exist, which is consistent with the results of Tong Xin [18]. When correcting gestational age to full term, Ramenghi [21] found that premature infants with focal white matter damage had cortical dysplasia by MR maturity score. Miller [22] showed that premature infants with focal white matter damage had myelination disorder by diffusion tensor imaging (DTI), even in children and adolescents; this abnormality still exists [23]. These results indicate that white matter damage in premature infants has an effect on the development of white matter. As a result, most children have mild cognitive impairment at a later stage [21]. Therefore, early and adequate intervention treatment for premature infants with white matter damage may help to improve their nerve development and alleviate symptoms.

\section{Conclusion}

In summary, focal white matter lesions in premature infants are predominantly located in the central region. The early stage is characterized by a hyperintense signal in DWI. Over time, the hyperintense signal in DWI gradually decreases, while the hyperintense signal in T1WI gradually increases. The ADC value showed that the range of white matter damage was larger than that shown by the conventional MRI sequence and could be used to evaluate the outcome of the lesion more accurately.

\section{Abbreviations}

PWMD (punctate white matter damage)

ADC(apparent diffusion coefficient)

FLAIR (fluid-attenuated inversion recovery)

PVL(periventricular leukomalacia )

\section{Declarations}


Ethics approval and consent to participate This study has been approved by the ethics committee of the Affiliated Hospital of Qingdao University.Informed consent was obtained from the parents of the infants.

Consent for publication The patient's parents have signed the written informed consent and allowed the publication of this article.

Availability of data and material All data generated or analysed during this study are included in this published article [and its supplementary information files].

Competing interests The authors declare that they have no competing interests.

Funding None.

Authors' contributions JZ was a major contributor in writing the manuscript.CC,CD,MM analyzed MRI image and measure $A D C$ value. $H L$ was in charge of statistical analysis. $X L$ was responsible for Article final verification and correspondence author.All authors read and approved the final manuscript.

Acknowledgements Not applicable.

\section{References}

1. Volpe JJ.Encephalopathy of prematurity includes neuronal abnormalities[J].Pediatrics,2005,116(1):221-225.

2. KINNEY HC. The encephalopathy of prematurity: one pediatric neuropathologist's perspective[J]. Seminars in pediatric neurology,2009,4(4):179-190.

3. Volpe JJ.Neurobiology of periventricular leukomalacia in the premature infant[J].PediatrRes,2001,50(5)553-562.

4. Maalouf EF,Duggan PJ,Counsell SJ,et al.Comparison of findings on cranial ultrasound and magnetic resonance imaging in preterm infants[J].Pediatrics,2001,107:719-727.

5. FU J, XUE X , CHEN L ,et al. Studies on the value of diffusion-weighted MR imaging in the early prediction of periventricular leukomalacia[J]. Journal of neuroimaging ,2009,1(1):13-18.

6. Bozzao A,DiPaolo A,Mazalezi C,et al. Diffusion-weighted MR imaging in the diagnosis of periventricular leukomalacia [J]. Eur Radio,2003,13:1571-1576.

7. Rutherford MA, Counsell S, Allsop J, et al. Diffusion-weighted magnetic resonance imaging in term perinatal brain injury: a comparison with site of lesion and time from birth [ $\mathrm{J}$ ]. Pediatrics, 2004,114 :1004-1014.

8. Mary A, Rutherford, Veena Supramaniam, et al. Magnetic resonance imaging of white matter diseases of prematurity[J]. Neuroradiology, 2010,52:505-521.

9. DYET LE, KENNEA N, COUNSELL SJ,et al. Natural history of brain lesions in extremely preterm infants studied with serial magnetic resonance imaging from birth and neurodevelopmental 
assessment[J]. Pediatrics: Official Publication of the American Academy of Pediatrics,2006,2(2):536548.

10. Inage $\mathrm{YW}$, Itoh $\mathrm{M}$, Takashima S.Correlation between cerebrovascular maturity and periventricular leukomalacia[J].Pediatr Neurol,2000,22(3):204-208.

11. Cornette LG, Tanner SF, Ramenghi LA,et al. Magnetic resonance imaging of the infant brain: anatomical characteristics and clinical significance of punctate lesions[J]. Arch Dis Child Fetal Neonatal Ed,2002,86: 171-177.

12. Roelants- van Rijn AM, van der Grond J, Stigter RH,et al.Cerebral structure and metabolism and longterm outcome in small- for- gestational- age preterm neonates[J].Pediatr Res, 2004,56:285.

13. Gomori JM,Grossman RI,Goldberg HI,et al.(1985)Intracranial hematomas:imaging by high-field MR[J]. Radiology 157:87-93.

14. Counsell J,Rutherford MA, Cowan FM, et al. Magnetic resonance imaging of preterm brain injury[J]. Arch Dis Child Fetal Neonatal ,2003,88:269-274.

15. Miller SP,Cozzio C,Goldstein RB,et al.Comparing the diagnosis of white matter injury in premature newborns with serial MR imaging and transfontanel ultrasonography findings[J].American Journal of Neuroradiology,2003,24:1661-1669.

16. Inder T, Huppi PS, Zientara GP, et al. Early detection of periventricular leukomalacia by diffusionweighted magnetic resonance imaging techniques[J]. Pediatric, 1999,134:631-634.

17. Feldman HM.Lee ES,Loe IN.et al,White matter microstructure on diffusion tensor imaging is associated with conventional magnetic resonance imaging findings and cognitive function in adolescents born preterm[J].Dev Med Child Nenrol,2012,54:809-814.

18. Tong $X$,Xue XD,Fu JH.Exploring the clinical significance of continuously measuring apparent diffusion coefficient values in the preterm infants with punctate white matter damage by applying diffusion weighted imaging[J].Chinese Journal of Pediatrics,2014,52 $277-281$. \In Chinese

19. Marin-Padilla M (1997) Developmental neuropathology and impact of perinatal brain damage: White matter lesions of the neocortex.[J] Neuro pathol Exp Neurol 56:219-235.

20. VAN KOOIJ BJ, DE VRIES LS, BALL G , et al . Neonatal tract-based spatial statistics findings and outcome in preterm infants[J]. A m J Neuroradiol,2012,33 (1): 188-194.

21. Ramenghi LA,Fumagalli $M$,Righini $A$, et al.Magnetic resonance imaging assessment of brain maturation in preterm neonate with punctuate white matter lesions[J] .Neuroradiology, 2007,49 (2):161-167.

22. Miller SP, Vigneron DB, Henry RG, et al.Serial quantitative diffusion tensor MRI of the premature brain development in newborns with and without injury. [J]Magn Reson Imaging,2002,16:621-632

23. EIKENES L,LOHAUGEN GC,BRUBAKK AM,e $t$ al.Young adults born preterm with very low birth weight demonstrate widespread white matter alterations on brain DTI[J ]. Neuroimage,2011,54 ( 3 ) 17741785. 


\section{Figures}
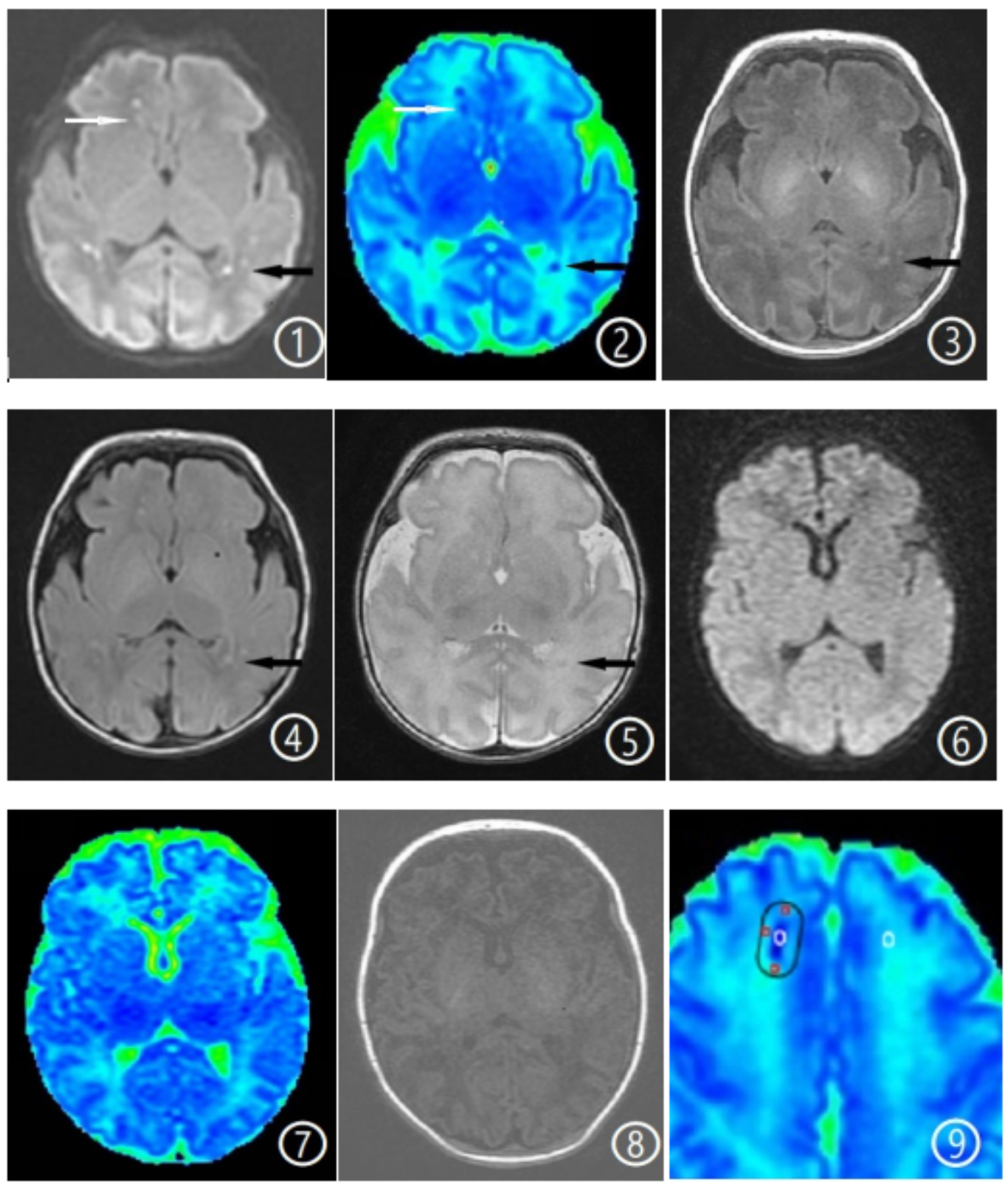

\section{Figure 1}

Fig 1, 2 As shown by the black arrow, the lesions showed a hyperintense signal in DWI and a decreased signal on $A D C$, and the range of some lesions on $A D C$ was slightly larger than that on DWI (as shown by the white arrow); Fig 3-5 The lesions showed a hyperintense signal in T1WI and FLAIR and a low signal on T2WI (indicated by the black arrow); Fig 6, 7 and 8 The abnormal signal on DWI, ADC and T1WI disappeared at the time of reexamination; Fig 9 The ADC value in the focus (white circle) of a child was $1.08 \times 10-3 \mathrm{~mm} 2 / \mathrm{s}$, the average $A D C$ value of three points (red circle) in the $5 \mathrm{~mm}$ range (black circle) around the lesion was $1.58 \times 10-3 \mathrm{~mm} 2 / \mathrm{s}$, and the $A D C$ value in the contralateral area of the lesion was $1.72 \times 10-3 \mathrm{~mm} 2 / \mathrm{s}$. 